\title{
El fraude en la ciencia: reflexiones a partir del caso Hwang
}

\author{
Por Emilio Delgado López-Cózar, Daniel Torres Salinas y Álvaro Roldán López
}

\begin{abstract}
Resumen: En 2006 el fraude cometido por Hwang en dos trabajos publicados en Science acerca de la investigación sobre células madre (stem cells) volvió a abrir el debate sobre las prácticas deshonestas en el mundo de la ciencia. A partir del mismo se reflexiona sobre las dimensiones del fraude en la ciencia y se analiza la singularidad del caso Hwang por su impacto científico, social y mediático como consecuencia de los mecanismos de persuasión sistematizados en la denominada Rutas de la mentira científica. Asimismo, se presentan las causas que conducen a la publicación de investigaciones fraudulentas, la ineficacia del arbitraje científico para detec-

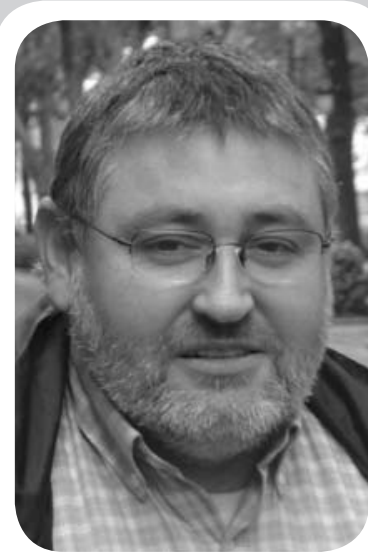

Emilio Delgado López-Cózar, es profesor de metodología de la investigación en la Facultad de Biblioteconomía y Documentación y miembro del grupo EC3 (Evaluación de la Ciencia y de la Comunicación Científica) de la Universidad Granada.

tarlas y los diversos mecanismos existentes para su gestión y erradicación.
\end{abstract}

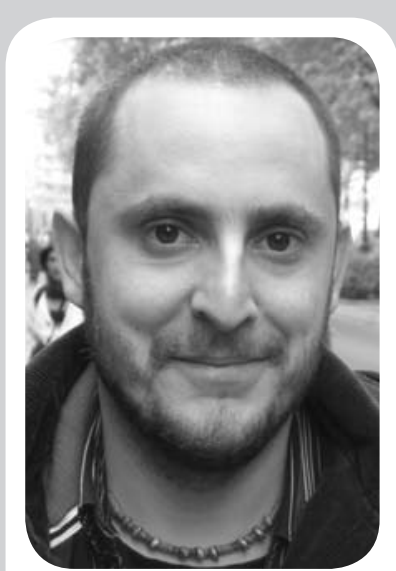

Daniel Torres Salinas, es investigador contratado del Centro de Investigación Médica Aplicada de la Universidad de Navarra y miembro del grupo EC3 (Evaluación de la Ciencia y de la Comunicación Científica) de la Universidad de Granada. En la actualidad realiza su tesis sobre la evaluación bibliométrica de instituciones médicas.

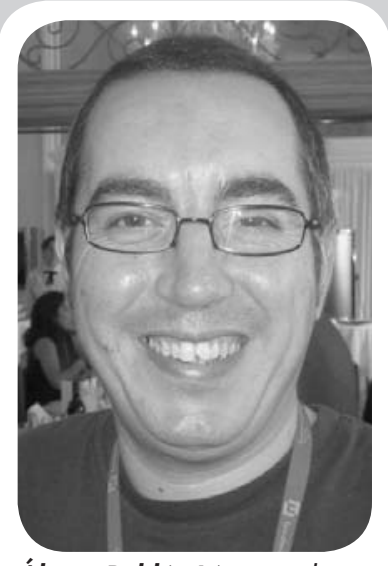

Álvaro Roldán López, es licenciado en documentación por la Universidad de Granada. Trabaja en el Fondo de Investigación Sanitaria, dependiente del Instituto de Salud Carlos III, donde una de sus principales misiones es gestionar el proceso de evaluación de proyectos de investigación. Además es el creador y el principal redactor de Bibliometria.com.

Palabras clave: Ética, Ciencia, Fraude científico, Deshonestidad científica, Publicación científica, Revisión por pares, Caso Hwang.

\section{Title: Fraud in science: reflections on the Hwang affair}

Abstract: In 2006 Hwang's fraudulent papers published in Science on stem cell research re-opened the debate on scientific misconduct. Taking this as a starting point, some reflections are put forward on the dimension of fraud in science. The singularity of Hwang's case, due to its scientific, social and media impact, is examined as a consequence of the persuasion mechanisms systematised in the so-called Routes of Scholarly Lying. Furthermore, the reasons for publishing fraudulent research, the inefficiency of peer review for detecting it, and the various mechanisms for managing and eradicating it, are presented.

Keywords: Ethics, Science, Scientific fraud, Scientific misconduct, Scholarly publishing, Peer review, Hwang affair.

Delgado-López-Cózar, Emilio; Torres-Salinas, Daniel; Roldán-López, Álvaro. "El fraude en la ciencia: reflexiones a partir del caso Hwang”. En: El profesional de la información, 2007, marzo-abril, v. 16, n. 2, pp. 143-150.

DOI: 10.3145/epi.2007.mar.07

\section{Introducción}

EL PASADO AÑO EL MUNDO DE LA CIENCIA se vio convulsionado por un auténtico terremoto causado, esta vez, no por un deslumbrante hallazgo sino por un monumental fraude científico. Un escándalo que ha traspasado los círculos científicos convirtiéndose en un fenómeno social que ha ge- nerado un importante debate público. Nos referimos al fraude cometido por Woo Suk Hwang que en 2004 anunció haber producido por primera vez en la historia células madre de embriones humanos clonados y, en 2005, haber extraído dichas células de la piel de pacientes enfermos, con lo que pueden usarse como fuente para regenerar todo tipo de tejidos, emplearse en trasplantes y en la curación de cualquier enfermedad degenerativa.

Los dos magnos descubrimientos fueron publicados en sendos artículos, requisito imprescindible para que la comunidad científica los aceptara y reconociera como ciertos. Vieron la luz en Science una de esas dos revistas que aparecen siempre con la vitola de máximo prestigio, con lo cual obtenían 
el máximo aval científico posible. Con ello Woo Suk Hwang no sólo se aseguraba la credibilidad científica sino que conseguiría la más alta difusión. Efectivamente, de forma inmediata obtuvo el reconocimiento de los científicos, fue aclamado en su país como un auténtico héroe nacional y despertó el entusiasmo de millones de enfermos.

Ahora que se conocen los detalles de la mentira y se han acallado los ecos mediáticos del caso entendemos que es buen momento para reflexionar sobre las implicaciones que el fraude tiene para la ciencia, para la comunidad científica que la sustenta y, muy especialmente, para el sistema de publicación científica como garante de la veracidad de la actividad investigadora y como filtro de sus impurezas.

Admitido que el fraude existe en la ciencia, seguramente para perplejidad del ciudadano de a pie que no podía siquiera imaginarlo, son varios los interrogantes que este suceso pone encima de la mesa, algunos de ellos bastante inquietantes. A saber:

- ¿Es éste un caso aislado o el fraude es un mal más extendido de lo que se piensa?, ¿cuáles son los tipos de fraude más frecuentes en ciencia?

- ¿Qué es lo que diferencia a este fraude de otros que ha habido?, ¿por qué ha adquirido tanta notoriedad?

- ¿Cómo puede ocurrir esto en el templo del saber que se supone inmaculado, altruista, desinteresado, volcado en la búsqueda del conocimiento y la verdad?, ¿cuáles son las causas o los factores que lo generan, promueven o potencian?

- ¿Existen medios para evitarlo e impedirlo?, ¿es la publicación un sello que garantiza la fiabilidad y validez de una investigación?, ¿pueden las revistas científicas con su sistema de evaluación por expertos "peer review" detectarlo y anularlo?

- ¿Cómo gestiona el sistema científico estos casos?, ¿existen protocolos de actuación?

- ¿Qué consecuencias tiene para la percepción de la ciencia por la sociedad, para la propia comunidad científica, para el sistema de publicación?

- ¿Qué medidas pueden ser adoptadas para evitar en el futuro casos parecidos?

\section{El tamaño del fraude científico}

El fraude en la ciencia no es algo nuevo. Siempre ha habido científicos que han engañado, como bien se ha documentado (Kobn, 1988; Trochio, 1993). Desde los comienzos de la ciencia moderna sabios tan egregios como Galileo, Newton, Mendel o Freud en ocasiones fueron poco honestos científicamente hablando. La deshonestidad científica, por tanto, no es sólo de ahora, pero lo que sí es novedoso es la proliferación de fraudes que han saltado a la palestra en los últimos años convirtiéndose en un mal más extendido de lo que se piensa. Lo que ocurre es que posee unas dimensiones desconocidas y difíciles de mensurar. Detectar el fraude y conseguir que aflore es bastante complicado debido a la imagen angelical y beatífica que la ciencia posee y la pérdida de prestigio que puede suponer para instituciones de investigación y revistas.

Más difícil es aún cuando las imposturas éticas son leves (incumplimiento de protocolos y reglas éticas en la investigación con ani- males o humanos, publicación prematura o duplicada, autoría ficticia, honorífica o fantasma, no declaración de conflictos de interés, falta de competencia, objetividad y rapidez en la evaluación de proyectos o revisión de manuscritos, omisión de citas a autores relevantes, y un largo etcétera). Sin embargo, al igual que nos enseñaba el catecismo a distinguir entre pecados capitales $y$ veniales, en ciencia hay que diferenciar los fraudes con mayúscula y con minúscula. Así, cuando se produce la invención (creación de datos o resultados registrándolos e informándolos como si fueran reales y ciertos), la falsificación (manipulación de materiales de investigación, equipos, procesos, o cambio u omisión de datos o resultados), o el plagio (apropiación de las ideas, resultados o palabras de otros autores sin ofrecerles el reconocimiento debido) el eco social y la denuncia pública está garantizado.

Diversos organismos como la Office of Research Integrity (ORI) recogen e investigan denuncias sobre estos fraudes con mayúsculas. Para el período 1994-2003 esta oficina recibió un total de 1.777 denuncias sobre posibles fraudes de las cuales 259 fueron tramitadas. El 59\% de estas investigaciones descubrieron algún tipo de mala conducta científica. Los resultados de la figura 1 ponen en evidencia cuáles son los tres grandes tipos de fraude en la investigación.

También el Committee on Publication Ethics (COPE), entre 1997 y 2004 tramitó 212 denuncias, 58 relativas a publicación duplicada o redundante, 26 a cuestiones de autoría, 25 de falta de ética en comités científicos, 22 por
"Detectar el fraude y conseguir que aflore es bastante
complicado debido a la pérdida de prestigio que puede
suponer para instituciones de investigación y revistas" 
informar inadecuadamente, 19 por fabricación y plagio, 15 por malas prácticas clínicas y 8 por conflictos de intereses (Kleinert, 2005). Otro indicador que nos puede permitir medir el tamaño del fraude es la retirada de trabajos por las propias revistas científicas. Según los datos del investigador Massimo Battaglia desde el año 1973 hasta 1999 se ha retractado un total de 387 artículos y en 321 de ellos se informó convenientemente a sus lectores por parte de las revistas (Battaglia, 2000). Aunque no se especifican las causas de las mismas, ya que algunas se deben a errores involuntarios, sí se constata un aumento del número de retractaciones acorde con el incremento en la cantidad de publicaciones. Otro análisis más preciso de las 395 retracciones de las revistas indexadas en Medline entre 1982 y 2002 revela como en 107 artículos (un 21\%) las causas de la retirada se debieron a casos importantes de deshonestidad científica (Nath, et al., 2006).

Aunque los fraudes con mayúsculas sean tremendamente explosivos y espectaculares, los que más dañan y corroen la ciencia son las deshonestidades más prosaicas, aquellas que son moneda de cambio en la ciencia. Porque si la misma lupa con la que se indagó al científico surcoreano se aplicara al resto quedaríamos sorpren- didos. ¿Quién no se ha enfrascado en problemas irrelevantes e insustanciales que consumen recursos? ¿quién no ha maquillado, cocinado y cuadrado a martillazos, si ha el caso, los datos para que ajusten a las hipótesis mantenidas?, ¿quién no ha cortado y copiado y ha tomado como suyas las ideas ajenas sin citar a su propietario?, ¿quién por envidia o competencia desleal o por otros motivos inconfesables no ha intentado obstaculizar, ocultar, minusvalorar o menospreciar el trabajo de los competidores?, ¿quién no ha firmado un trabajo sin haber participado suficientemente en él?, ¿quién no ha inflado artificialmente su número de publicaciones repitiendo lo comunicado y desgajando en pequeñas dosis el cuerpo central de la investigación? No todos los científicos podríamos responder de forma tajante y negativa a estas preguntas.

\section{El impacto del caso Hwang: la ruta de la mentira científica}

Lo que diferencia y singulariza el caso Hwang de otros que se han producido en la ciencia es su alta repercusión e impacto producidos en tres niveles: científico, mediático y social.

- Científico: el gran número de personas implicadas. Estos expe-

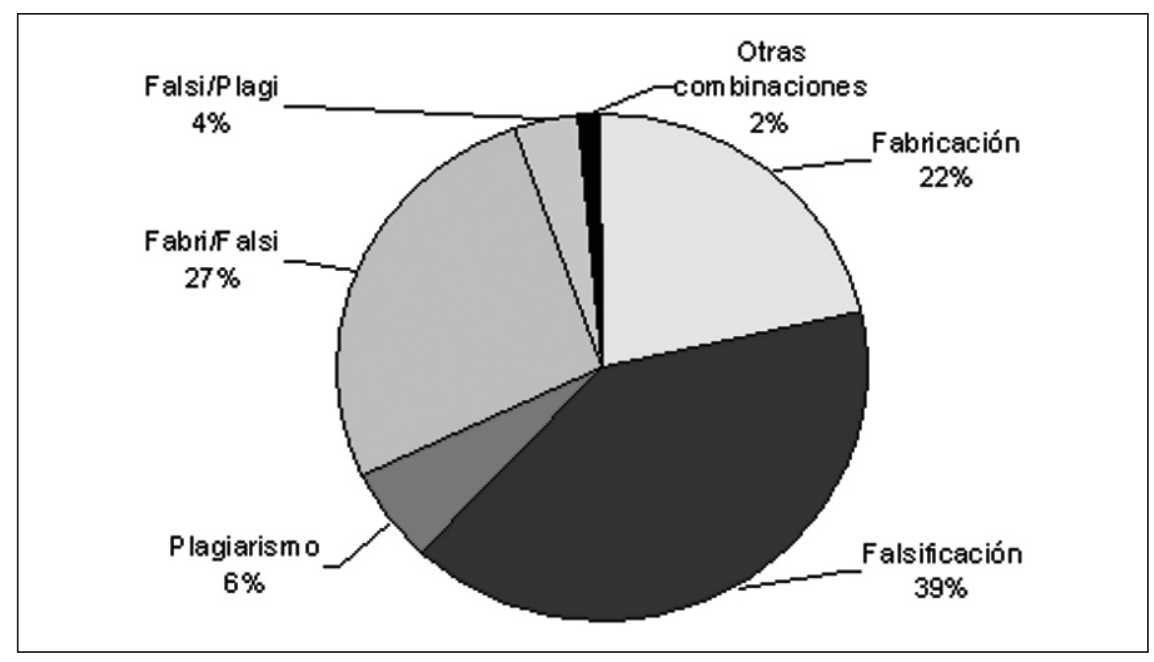

Figura 1. Los fraudes con mayúsculas

rimentos no se hacen en la soledad del laboratorio: los trabajos fueron firmados por 30 investigadores (entre ellos dos de los científicos estadounidenses más prestigiosos en la materia) y por 10 universidades y centros de investigación. Además, han podido ser leídos por todos los especialistas del mundo. Esto quiere decir que el fraude ni fue detectado antes por los evaluadores de Science, ni después por toda la comunidad científica. Esta situación, la incapacidad para detectar una mentira, es lo realmente lesivo para la ciencia y el sistema de publicación científica.

- Mediático: la noticia abrió noticiarios, figuró en las portadas de los periódicos y fue objeto de amplios reportajes en revistas de todo el mundo. Los periodistas necesitan noticias que vender. La creación de la vida, su manipulación (la biotecnología), la curación de enfermedades comunes (diabetes, parkinson, alzheimer, etc.) son temas de alto impacto social, capaces de vender y de atraer la atención de toda la sociedad desde el estamento político hasta el religioso. Los grandes descubrimientos siempre han atraído las miradas de todo el mundo, pero es la primera ocasión en que un engaño sustituye a los grandes avances en las columnas de actualidad científica de los principales periódicos.

- Social: la gran implicación de la ciencia en la sociedad. Desde hace años sabíamos que la ciencia era el motor de progreso de la sociedad. Pero este caso ha ido todavía más allá, por cuanto demuestra que la ciencia puede trasmutarse en un fenómeno social de masas. Woo Suk Hwang se convirtió en una estrella mediática; cual deportista galáctico o estrella de rock, poseía su club de fans con más de 15.000 seguidores, muchos de ellos mujeres dispuestas a ceder sus óvulos. En 
Corea era considerado un héroe nacional, de hecho el gobierno de Seúl lo había designado "científico supremo” de la nación. De nuevo, la ciencia al servicio del poder.

Este impacto del caso Hwang en tres escenarios diferentes se explica por lo que podríamos denominar la ruta de la mentira científica que ilustra espléndidamente los mecanismos de propagación de las grandes mentiras científicas a través de un verdadero ejercicio persuasivo que tendría las siguientes escalas:

- Persuasión científica: primero se trata de convencer a los científicos: la publicación es el elemento clave, cuanto mayor prestigio tenga ésta mejor para conseguirlo. Primero se publica en las revistas internacionales de mayor impacto de la especialidad; si se consigue escalar a Science o Nature mejor que mejor (aunque para eso hay que asociarse con científicos del centro de avance científico, normalmente anglosajones). Una vez encaramado a dicho puesto, el efecto Mateo (quien más tiene, más recibe) empezará a dar sus réditos.

- Persuasión de los media: convencer a los periodistas: el aval científico es la llave para alcanzarlo. En los medios de comunicación tradicionales y en internet se incluyen más y más noticias médicas, con la premisa de que la salud vende. Muchas de estas noticias son de rigurosa actualidad científica, pues acaban de ver la luz en revistas de reconocido prestigio. Internet, además, ha facilitado la comunicación entre dos corrientes de información que antes apenas tenían contactos: los investigadores y sus resultados publicados, por un lado, y los medios que los difunden y los convierten en noticia para el gran público, por otro. Sin entrar por ahora en la calidad de esta información, existe un fenómeno que llama la atención del observador: la uniformidad del discurso, tanto en lo que respecta a las fuentes informantes como a los mensajes que se difunden. ¿Por qué los medios se hacen eco generalizado y preferente de los estudios de ciertas revistas o instituciones?, por qué el mensaje es, a menudo, tan uniforme? Un papel importante al respecto lo juegan las press releases (notas de prensa) elaboradas por las grandes revistas científicas, donde el descubrimiento científico se codifica en noticia de carácter general, en ocasiones omitiéndose las limitaciones de los estudios, magnificándose o malinterpretándose los resultados (Woloshin; Schwartz, 2002).

- Persuasión del gran público: de ello ya se encargan los periodistas y los especialistas en divulgación científica. Lo hacen idealizando la labor del investigador y magnificando los potenciales efectos de sus descubrimientos en la sociedad. Cuanto más excitante sea la noticia mayor es el tiempo invertido en el medio, cuanto más retórica y grandilocuente sea la redacción de la noticia mayor será el número de lectores. Como ejemplo proponemos el fragmento de un texto publicado en la versión electrónica de El mundo (Jiménez, 2005) que contrasta llamativamente con el escepticismo y la sobriedad propios de las publicaciones científicas.

- Persuasión del mecenas: convencer a los políticos, gestores y administradores públicos y a las empresas para que proporcionen la financiación adecuada para la investigación y para el medro en la carrera personal. Cae por su propio peso: el clamor social lo hace imprescindible.

La mentira científica se convierte en un complicado laberinto persuasivo cuyo descubrimiento suele costar un alto precio.

\section{En busca de las causas del fraude}

Si bien las palabras mentira, deshonestidad, fraude parecen intrínsecamente incompatibles con la actividad científica, no debemos olvidar que la ciencia la hacen personas de carne y hueso y que la condición humana es voluble. Los científicos, como seres sociales que son, no son distintos a los deportistas, políticos, periodistas o abogados. En la ciencia, como en cualquier actividad social, puede darse el fraude y la corrupción. ¿Acaso la naturaleza de la acción de Hwang es distinta al dopaje de un deportista o a la corrupción de un político?

Si la semilla del fraude se encuentra en la condición humana del científico, el abono hay que buscarlo en diversos factores que aportan nutrientes para que las malas prácticas florezcan. Cuando ellos se conjugan el arte de la ciencia da lugar a la ciencia del engaño que puede atrapar a aquellos científicos que se encuentren más predispuestos. Estos factores son los siguientes:

- La búsqueda del reconocimiento. Los hechos científicos sólo son tales cuando son reconocidos por la comunidad científica. La fama, la gloria, ser el primero, el anhelo de durar, de quedar para la posteridad son componentes básicos del quehacer científico. Hoy día son los engranajes que mueven el motor de la ciencia. El reconocimiento llegará con rapidez a través la originalidad y la prioridad. Evidentemente además este reconocimiento es intercambiable por los recursos económicos que permitirán en el futuro seguir aumentando el prestigio científico y social. En definitiva lo supone todo en la carrera de un científico: financiación, los mejores cargos institucionales, poder sobre un equipo, etc.

- La competencia globalizada. La competitividad es mayor que 


\section{Extracto de la noticia aparecida en El mundo (3 de agosto de 2005):}

"(...) En el laboratorio de la Universidad de Seúl donde se está transformando la forma en la que la vida es creada (...) Hwang y sus cerca de 40 colaboradores, la mayoría jóvenes científicos coreanos que le veneran, trabajan siete días a la semana en jornadas de 12 horas (...) está convencido de estar en plena misión por salvar al mundo de las enfermedades incurables del siglo XXI.

Ése es, al menos, el sueño declarado este hombre discreto y trabajador de 52 años. Quiere devolver la memoria a los enfermos de alzheimer, liberar a millones de diabéticos de la insulina o lograr que las personas con fracturas de la espina dorsal puedan volver a caminar. Para ello, asegura, la clonación ofrece oportunidades jamás imaginadas.

La reanudación de las investigaciones se ha hecho de forma discreta, pero el próximo experimento del científico promete ser una nueva bomba.

En el proceso ha sido creada una nueva vida, en sus fases iniciales, sin la intervención sexual de dos personas. Adán y Eva, los principios básicos de la reproducción humana, transformados para siempre.

Las dificultades económicas le obligaron a esforzarse más que nadie: su única oportunidad estaba en ser el mejor para lograr ayudas oficiales y fue finalmente una beca de la Universidad de Seúl la que le sitúo camino de lograr su sueño.

Nada más publicar su trabajo en Science, el doctor Hwang tuvo que defenderse de la acusación de que las donantes eran estudiantes que estaban realizando tesis doctorales bajo su supervisión presionadas para participar en el experimento. La polémica fue aprovechada por los opositores del investigador para tratar de detener su trabajo y desprestigiar su nombre.

Nadie que haya visto trabajar a Hwang, y desde luego nadie que le conozca, puede tener duda de que detrás de sus experimentos sólo se esconde el sueño de mejorar la vida de millones de enfermos. La fama y el dinero no parecen estar entre sus motivaciones. Pero incluso sus seguidores mantienen la preocupación de que sus avances en la clonación puedan ser utilizados por otras personas con menos escrúpulos y con otros fines.

(...) Ellos son la prueba de que, independientemente de que uno piense que estamos ante un nuevo Frankenstein o el próximo Nobel, ya no hay marcha atrás posible. La forma en la que la vida puede ser creada nunca volverá a ser lo que fue..."

\section{http://www.elmundo.es/elmundo/2005/08/03/ciencia/1 123078892.html}

nunca: hay más científicos y en más lugares del mundo. La fuerte rivalidad entre ellos o grupos de investigación, se convierte en tensa pugna entre unos y otros por llegar antes y más lejos. Esta feroz competencia se acrecienta con la masificación actual del plantel de científicos conformado por becarios de investigación, profesores, técnicos, ayudantes, colaboradores, etc., que han modificado la profesión y sus va- lores. Esta situación genera un gran número de profesionales que no aceptan las reglas éticas del juego científico, bien por desconocimiento, falta de formación o por la facilidad para obtener el beneficio rápido.

- La presión por los resultados. Los científicos deben producir resultados inmediatos. Las instituciones donde trabajan así se lo exigen. Se les juzgará precisamente por ello. La presión por publici- tarlos es tremenda: los científicos perecen por publicar, porque hacerlo significa poder investigar. Quien no genera resultados y no los transforma en publicaciones,

\section{"Las instituciones donde trabajan los científicos les exigen resultados inmediatos"}


que son los productos más tangibles de la ciencia, no será nada ni nadie en el cosmos científico.

- La ciencia como empresa. Hoy está más claro que nunca que los beneficios de las empresas dependen de los hallazgos científicos que son capaces de generar. La ciencia cotiza en bolsa, y esto añade fuertes presiones. Es paradigmático en este tema la presión que ejercen las grandes multinacionales farmacéuticas sobre los ensayos clínicos donde el interés de la industria no siempre se corresponde directamente con la prioridad de la ciencia (Friedman, 2004). Los ensayos clínicos rara vez producen resultados desfavorables a los intereses empresariales ya que su publicación supone una magnífica publicidad para las farmacéuticas y una fuente de financiación extra para las revistas científicas.

Con todos estos ingredientes debidamente conjuntados no es difícil explicarse el caso Hwang. Un científico con mucha ambición y pocos escrúpulos que sucumbe a la tentación de suprimir, cambiar e inventar datos para ver cumplidos sus sueños.

\section{Desmontando los mitos}

Es hora de desmontar algunos mitos sólidamente instalados en las
"No existen medios infalibles que puedan impedir el fraude, ni la publicación es un sello que garantice la fiabilidad y validez de una investigación"

conciencias científicas: ni existen medios infalibles que puedan impedir que el fraude se produzca, ni la publicación por sí sola es un sello que garantice la fiabilidad y validez de una investigación, ni el sistema de evaluación por expertos empleado tanto por las agencias evaluación como por las revistas es capaz de detectarlo y neutralizarlo. Básicamente por dos razones.

En primer lugar, porque el edificio científico se asienta sobre un pilar axiomático que es falseable: se basa en la buena voluntad de los científicos; la honestidad se presupone; es inconcebible que un científico premeditadamente esté dispuesto a mentir. Por consiguiente, si alguien quiere mentir lo hará. Y como no cabe en la mente de la comunidad científica que esto ocurra, la guardia estará permanentemente bajada. En segundo lugar, porque el sistema de alerta que emplea la ciencia para contrastar la verosimilitud y veracidad de un descubri- miento se aplica en muy pocos casos. La replicación y contrastación de la investigación por otros científicos, que es la auténtica máquina de la verdad, es impracticable dado el volumen actual que ha adquirido la ciencia.

Aunque supuesta y teóricamente las investigaciones pueden ser reproducidas, la sección metodológica de un trabajo ofrece los detalles que lo hacen factible, esto no se lleva a efecto más que con los grandes descubrimientos. Pero es que, además, la verificación de todos los resultados de investigación publicados es materialmente imposible: ¿qué tiempo requeriría esta tarea?, ¿disponen de él los revisores que deben valorar los trabajos?, ¿poseen el mismo nivel de competencia, conocimientos, habilidades técnicas y el equipamiento necesario para hacerlo?, ¿están libres de intereses los propios revisores para que se les pueda confiar los trabajos de sus potenciales competidores? Evidentemente no. Pero es que si se pretendiera hacerlo la ciencia iría al colapso. Por consiguiente, se parte del principio de confianza y de la buena fe al que antes aludíamos: si los científicos declaran haber hecho algo, se cree en ellos y se da por cierto.

El caso de Hwang nos enseña, de nuevo, como ocurrió en anteriores sucesos, que el fraude fue desta-

\section{Suscripciones}

Renovar (o comenzar) la suscripción a "El profesional de la información" es ágil y sencillo.

Usted puede gestionar online su suscripción conectándose a esta web: http://www.elprofesionaldelainformacion.com/suscripciones.html Si lo desea puede comunicar con nosotros dirigiéndose a: suscripciones@elprofesionaldelainformacion.com 
pado por los propios colaboradores o por personas ajenas a la investigación. Nunca fue detectado a tiempo por el sistema de evaluación por expertos. Debe quedar claro que el arbitraje científico no sólo no es capaz de descubrir el fraude sino que ni siquiera puede garantizar la fiabilidad y validez de los resultados. Muchas son las experiencias que han evidenciado la verdad de esta afirmación (Darsee, Slutsky, Schön, etc.), muchos los experimentos que han demostrado la inoperancia del sistema (Jurdant, 2003; Peters; Ceci, 1982; Epstein, 1990; Campanario, 1995, 2002) y muchas más las investigaciones que han puesto al descubierto los defectos del arbitraje científico (Armstrong, 1997).

Si el arbitraje es lento, costoso, despilfarrador del tiempo de la comunidad científica, subjetivo, propenso al sesgo, sin capacidad para evitar los abusos de editores o revisores, no puede garantizar la fiabilidad y validez de los trabajos, es incapaz de detectar la fabricación, falsificación, el plagio y el resto de deshonestidades científicas, ¿para qué sirve entonces?, ¿por qué lo seguimos utilizando? Las únicas certezas que poseemos es que este sistema sirve para reducir la avalancha de información, para mejorar la redacción, para enseñar a los autores a presentar sus trabajos de acuerdo con los cánones del método científico y para seleccionar algunos materiales buenos y rechazar muchos malos. Sólo podemos decir que cuanto más lo usamos menos nos gusta, pero cuando menos lo usamos más lo echamos en falta. Mayoritariamente se considera que, al igual que ocurre con la democracia, es el menos malo de los sistemas. Un sistema que ya es objeto de estudio con derecho propio como lo pone de manifiesto la celebración de los congresos internaciones sobre peer review en publicaciones biomédicas organizados por JAMA y el BMJ Publishing Group.

\section{La lucha contra el fraude}

Como consecuencia del panorama descrito en apartados anteriores es lógico que surgieran agentes y propuestas dispuestos a acabar con el fraude o al menos a evitarlo. Una forma de prevención bastante extendida es la emisión de normas y códigos. Este tipo de documentos pretenden advertir sobre las principales malas conductas, sus consecuencias y las posibles medidas y sanciones en caso de incurrir en ellas, así como detallados protocolos de actuación de los diferentes agentes implicados; predominan en todo tipo de instituciones y sociedades. Han sido emitidos por los organismos de financiación de la investigación, las universidades y centros de investigación, las sociedades científicas y asociaciones profesionales, las sociedades de edición científica y las propias revistas.

Pero quizás más eficaces, o al menos más visibles en su labor, se han mostrado diversas instituciones cuya misión es la identificación, gestión y difusión de los casos de deshonestidad científica. Los primeros países en instituir estos centros, como casi siempre ocurre, fueron los anglosajones y los nórdicos. Probablemente la más popular de todas sea la US Office of Research Integrity de los EUA que investiga los casos de fraude que llegan a sus oficinas en forma de alegaciones. Su actividad queda reflejada en sus informes de carácter anual donde además se publican las fichas con breves descripciones de los casos detectados. Un cometido similar desempeña el Committee on Publication Ethics del Reino Unido que reúne desde 1997 a diversos editores de revistas científicas, y también publica informes y ofrece acceso público a los casos que ha gestionado. Instituciones de este tipo han surgido en otros países como el Norwegian National Committee for the Evaluation of
Dishonesty in Health Research o el Danish Committee on Scientific Dishonesty. La mera existencia de organismos de esta naturaleza constata y ratifica el fraude y los comportamientos deshonestos como una práctica frecuente que merece una atención especial. Gracias a su labor los científicos son obligados en ocasiones a la retractación de sus trabajos, la inhabilitación para publicar en las revistas en que hayan visto la luz los trabajos, la suspensión de la financiación otorgada, si fuera el caso, y la inhabilitación futura para recibir fondos. Los casos más graves conllevan la expulsión de los investigadores de sus centros de trabajo y la persecución judicial de sus delitos. Sin embargo la mayoría de las veces en que se destapan, son resueltos sin luz ni taquígrafos en el seno de la institución donde trabajan los investigadores y no suelen tener mayores consecuencias.

\section{Conclusiones}

Admitiendo como cierta la tesis de que las conductas deshonestas en la ciencia están más extendidas de lo que oficialmente se reconoce y socialmente se admite, convendría adoptar medidas que intentaran paliar esta situación. Éstas podrían ser algunas de ellas:

- Como en cualquier tipo de enfermedad sea biológica o social la mejor actuación contra el fraude es prevenir antes que curar. Y para ello, lo mejor sería crear condiciones que favorezcan una investigación responsable y ética, promoviendo un entorno que predisponga a comportamientos éticos y destierre las desviaciones. Esto afecta tanto a la financiación de la investigación (impidiendo que los temas más sensibles y de mayor repercusión social no estén dominados exclusivamente por la industria privada), como al sistema de evaluación y de reconocimiento 
científico y académico, que no debe pivotar tan exclusivamente sobre los resultados y sobre la hiperproducción de publicaciones, como ocurre hoy día.

- Se debería institucionalizar un sistema de gestión del fraude científico en la línea de lo que ya se viene haciendo: crear comités ad hoc tanto en las instituciones financiadoras, en las universidades y centros de investigación, como en las publicaciones científicas. Asimismo, la persecución de los científicos deshonestos debe ser resuelta. La transparencia absoluta con una amplia difusión de los defraudadores debe combinarse con el establecimiento de duras sanciones.

- Respecto al arbitraje científico, piedra angular sobre la que descansa el sistema de evaluación de la ciencia, deberíamos aprovechar las posibilidades tecnológicas que ofrece la sociedad de la información. Sirviéndonos de las facilidades que ofrece internet convendría abrir al máximo el proceso de evaluación incentivando a los lectores a que puedan replicar con libertad los trabajos publicados y colocar como material adjunto todos los datos y registros generados en el proceso de investigación y pedir a los autores declaraciones sobre cuál ha sido su contribución a la investigación. Estas medidas no impedirán el fraude, pero lo dificultarán.

\section{Bibliografía}

Armstrong, J. S. "Peer review for journals: evidence on quality control, fairness, and innovation". En: Science and engineering ethics, 1997, v. 3, pp. 63-84.

Battaglia, M. Retractions 1966-99. Consultado en: 23-11-06.

http://pages2.inrete.it/mbiomed/rtrx6699.htm

Campanario, J. M. “Commentary: on influential books and journal articles initially rejected because negative referees' evaluations". En: Science communication, 1995 , v. 16, pp. 304-325.

Campanario, J. M. "El sistema de revisión por expertos (peer review): muchos problemas y pocas soluciones". En: Revista española de documentación científica, 2002, v. 25, n. 3, pp. 166-184.

Epstein, W. M. "Confirmational response bias among social work journals". En: Science, technology and human values, 1990, v. 15, pp. 9-38.

Friedman, L.; Richter, E. D. "Relationship between conflicts of interest and research". En: Journal of general and internal medicine, 2004, v. 19 , pp. 51-56.

Jiménez, D. Entrevista al científico surcoreano Woo Suk Hwang, creador de 'Snuppy'. Consultado en: 18-11-06.

http://www.elmundo.es/elmundo/2005/08/03/ ciencia/1123078892.html

Jurdant, B. Imposturas científicas: los malen- tendidos del caso Sokal. Cátedra: Madrid, 2003, Isbn 84-376-2079-1.

Kleinert, S.; Theobald, J.; Wager, E.; Godlee, F. For which cases of suspected misconduct do editors seek advice? An observational study of all cases submitted to COPE. Consultado en: 18-11-06. http://www.ama-assn.org/public/peer/abstracts. html

Kobn, A. False prophets: fraud and error in science and medicine. New York: Basil Blackwe11, 1988, Isbn 0-6311-6237-2.

Nath, S.; Marcus, S.; Druss, B. G. "Retractio$\mathrm{ns}$ in the research literature: misconduct or mistakes?". En: The medical journal of Australia, 2006 , v. 185 , n. 3 , pp. $152-154$

Peters, D. S.; Ceci, S. J. "Peer-review practices of psychological journals: the fate of published articles, submitted again". En: The behavioral artd brain sciences, 1982, v. 5, pp. 187-195.

Trocchio, F. Las mentiras de la ciencia. Madrid: Alianza, 1997, Isbn 84-206-3988-5.

Woloshin, S.; Schwartz, L. "Translating research into news". En: JAMA, 2002, v. 287, n. 21, pp. 2.856-2.858

Emilio Delgado López-Cózar, Departamento de Biblioteconomía y Documentación. Universidad de Granada.

edelgado@ugr.es

Daniel Torres Salinas, Centro para la Investigación Médica Aplicada. Universidad de Navarra. torressalinas@gmail.com

Álvaro Roldán López, Instituto de Salud Carlos III. aroldan@ISCIII.ES

\section{IraLIS}

Por iniciativa de esta revista EPI, el Directorio EXIT, el repositorio E-LIS y la Univ. Politécnica de Valencia, en febrero de 2007 se puso en marcha el International Registry for Authors in Library and Information Science con el fin de estandarizar las firmas e identificar así mejor a cada autor, en especial a los de habla hispana. IraLIS también pretende que las bases internacionales citen adecuadamente a los autores.

A partir del próximo número (vol. 16, n. 3, mayo-junio 2007) EPI aplicará el formato iralis a los nombres de todos los autores firmantes. http://www.iralis.org/ 